\title{
Habilidade de atenção compartilhada em sujeitos com transtornos do espectro autístico***
}

\author{
Joint attention ability in children with autistic spectrum disorders
}

\author{
Camila Gioconda de Lima e Menezes* \\ Jacy Perissinoto**
}

*Fonoaudióloga. Especialização em Desenvolvimento de Linguagem e suas Alterações pela Universidade de São Paulo. Mestre em Ciências pelo Programa de Pós-Graduação em Distúrbios da Comunicação Humana do Departamento de Fonoaudiologia da Universidade Federal de São Paulo. Endereço: Rua Botucatu, 802 - São Paulo - SP - CEP 04023-900 (cagimenezes@yahoo.com.br).

**Fonoaudióloga. Doutora do Programa de Pós-Graduação em Distúrbios da Comunicação Humana do Departamento de Fonoaudiologia da Universidade Federal de São Paulo. Pós-Doutorado - Paris (Sorbone).

***Trabalho Realizado na Universidade Federal de São Paulo.

Artigo Original de Pesquisa

Artigo Submetido a Avaliação por Pares

Conflito de Interesse: não

Recebido em 23.11.2007.

Revisado em 30.03.2008; 30.06.2008; 29.07.2008

Aceito para Publicação em 21.10.2008.

\begin{abstract}
Background: joint attention in children with autistic spectrum disorders. Aim: to evaluate joint attention ability in subjects with autistic spectrum disorders in different contexts and with different interlocutors. Method: twenty subjects with ages between four and twelve years, diagnosed with autism or asperger syndrome (DSM-IV-TR, 2002) by a multidisciplinary team, with no associated disorders, and who were enrolled in language therapy for at least six months were assessed. The Symbolic Maturity Assessment Test was used to evaluate joint attention behaviors. An adaptation that included the Semi-Structured Play situations was carried out for this research. For all of the studied situations (free play; semi-structured play with speech and language therapist and the caregiver; imitation) the following joint attention behaviors were observed: alternate, point, show (by child's initiative or in response to adult's action) and look at adult's action (response behavior). The last included the differentiation of the ways by which the adult drew the child's attention (talking; gesturing; or both). Results: the different situations changed the way these subjects shared attention. The adult's intervention proved to be effective in triggering an increase in the occurrence of joint attention behaviors, mainly in response to the adult. No differences were found between interlocutors (speech therapist and mother) in semi-structured situations, probably because both interlocutors synchronized their behaviors with the children. Conclusion: the evaluation of joint attention behaviors in play contexts was effective and the interference of a known adult was reflected in the increase of the occurrence of these behaviors in semi-structured and imitation situations.
\end{abstract}

Key Words: Autism; Asperger Syndrome; Autism Spectrum Disorders; Attention; Language.

\section{Resumo}

Tema: atenção compartilhada em sujeitos do espectro autístico. Objetivo: avaliar a habilidade de atenção compartilhada em sujeitos com transtornos do espectro autístico em diferentes contextos e com diferentes interlocutores. Método: foram avaliados vinte sujeitos com idades entre quatro e doze anos, com autismo infantil ou síndrome de asperger (DSM-IV-TR, 2002) sem outros transtornos, diagnosticados por equipe multidisciplinar, que estavam em terapia fonoaudiológica há pelo menos seis meses. Para avaliação dos comportamentos de atenção compartilhada foram utilizados os materiais e procedimentos da Avaliação da Maturidade Simbólica, além de uma adaptação feita para esta pesquisa que incluiu as situações SemiDirigidas com interlocutores familiares. Para as situações estudadas (brincadeira livre; semi-dirigidas com terapeuta e cuidador; imitação), foram observados os comportamentos de atenção compartilhada alternar, apontar, mostrar (por iniciativa ou resposta da criança) e olhar para a ação do adulto (sempre por resposta da criança). Este último incluiu a diferenciação do meio utilizado pelo adulto para chamar a atenção da criança (fala; gestos; ou, ambos). Resultados: as diferentes situações modificaram a forma como estes sujeitos compartilharam a atenção. A intervenção de um adulto aumentou a ocorrência dos comportamentos de atenção compartilhada principalmente em Resposta. Não foram observadas diferenças na intervenção dos diferentes interlocutores (fonoaudiólogo e mãe) nas situações semi-dirigidas, provavelmente porque ambos sincronizaram seus comportamentos com as crianças. Conclusão: a avaliação da atenção compartilhada em contexto de brincadeira foi eficaz e a intervenção do adulto refletiu no aumento destes comportamentos nas situações semi-dirigidas e de imitação.

Palavras-Chave: Autismo Infantil; Síndrome de Asperger; Transtornos do Espectro Autístico; Atenção; Linguagem.

Referenciar este material como: 


\section{Introdução}

Os quadros do espectro autístico são bastante heterogêneos, variam em sua gravidade, porém mantêm em comum os prejuízos nos aspectos sociais, da comunicação e dos comportamentos e interesses ${ }^{1-2}$.

As dificuldades relacionam-se aos aspectos verbais e não-verbais. Estes últimos têm sido considerados importantes - especialmente a atenção compartilhada - para a identificação precoce e o desenvolvimento social e de linguagem das crianças do espectro autístico ${ }^{3-4}$.

No desenvolvimento típico, a atenção compartilhada aparece no final do primeiro ano de vida e envolve direcionar a atenção de um parceiro comunicativo com a intenção de dividir uma situação ${ }^{5}$.

A relação precoce da criança na díade com o adulto determinará o sucesso em seu desenvolvimento social e de linguagem. $\mathrm{O}$ adulto aproveitará suas pistas para introduzir as referências sobre o mundo, proporcionará à criança compreender a intenção comunicativa e facilitará o compartilhamento da atenção ${ }^{6}$.

A atenção compartilhada tem sido considerada como um marcador precoce para a identificação e a intervenção dos quadros do espectro autístico, visto que é precursora para o desenvolvimento de linguagem ${ }^{7-9}$. Os comportamentos de atenção compartilhada podem ser classificados em sua ocorrência por iniciativa da criança ou em resposta à ação do adulto, sendo que este último é o mais freqüente nesses quadros justamente devido às dificuldades que estes sujeitos possuem no uso e compreensão da intenção comunicativa ${ }^{10-13}$.

Considerando-se os prejuízos destes indivíduos nesta habilidade, hipotetiza-se que esta seja influenciada pelo contexto situacional e de interação.

Desta forma, os objetivos desse estudo foram verificar a habilidade de atenção compartilhada nas situações de brincadeira livre, semi-dirigidas e imitação, em crianças com transtornos do espectro autístico, a partir da intervenção de diferentes interlocutores.

\section{Método}

Sujeitos

Foram avaliadas 20 crianças, 4 meninas e 16 meninos com idade média de 9:4 anos, com diagnóstico multidisciplinar de autismo infantil ou síndrome de asperger ${ }^{14-15}$. Foi considerado como critério de inclusão que todas as crianças estivessem em terapia fonoaudiológica há pelo menos seis meses, e como critérios de exclusão a presença de malformações e/ou síndromes genéticas associadas, alterações neurológicas, deficiências física, auditiva ou visual. Apenas, uma criança não estava inserida em algum programa educacional. Os procedimentos iniciaram após aprovação do Comitê de Ética em Pesquisa e da assinatura pelos responsáveis do Termo de Consentimento Livre e Esclarecido (CEP/ Unifesp número 807/05).

As crianças eram provenientes do Laboratório de Investigação Fonoaudiológica da Linguagem nos Transtornos Globais do Desenvolvimento e do Centro de Referência da Infância e Adolescência, dos Departamentos de Fonoaudiologia e Psiquiatria da Unifesp.

\section{Material}

Foi utilizada a Avaliação da Maturidade Simbólica ${ }^{16-17}$, cujo objetivo é avaliar o desempenho cognitivo de crianças com alterações no desenvolvimento de linguagem, através de brinquedos mais e menos representativos. Nesta pesquisa, o material foi utilizado com o objetivo de verificar a habilidade de atenção compartilhada.

Os objetos da brincadeira livre são apresentados à criança em uma caixa: telefone de brinquedo, uma bola grande, bonecas e animais de tecido ou plástico, alguns veículos pequenos, utensílios de cozinha, mamadeira, um cobertor e um travesseiro pequenos, um colar longo, blocos e copos seriados, ábaco e dois brinquedos mecânicos de corda.

Os objetos da imitação foram apresentados à criança conforme as ações: copo; escova de dente; colher; boneca; sabonete; flor; sapo; avião; chapéu; carro; banana; berço; banheira; babador; cobertor; livro e toalha.

\section{Procedimento}

Originalmente, a Avaliação da Maturidade Simbólica propõe as situações de brincadeira livre e imitação ${ }^{16-17}$. Para este estudo, o procedimento foi adaptado, acrescentando-se uma filmagem interativa com o terapeuta/fonoaudiólogo e outra com o cuidador/mãe, denominadas situações semidirigidas, para observação de possíveis modificações na atenção compartilhada pela intervenção de diferentes interlocutores.

A avaliação foi dividida em duas sessões, com duração aproximada de 45 minutos e intervalo entre 
elas de pelo menos 15 dias, para diminuir possíveis influências da sessão inicial no próximo contato com o mesmo material.

As sessões foram filmadas individualmente, com a presença de um adulto familiar (fonoaudióloga e/ou mãe), para evitar que a interação com uma pessoa não conhecida interferisse no desempenho.

Primeira sessão

Situação 1 - brincadeira livre

Foi apresentada à criança uma caixa de brinquedos com a instrução: "Você pode brincar com estes brinquedos do jeito que você quiser". O adulto só interagiu quando a criança iniciou o contato ou para chamar a atenção caso ela permanecesse muito tempo explorando um único objeto, sem sugerir qualquer atividade.

Situação 2 - semi-dirigida com terapeuta fonoaudiólogo

Foi utilizado o mesmo material da brincadeira livre, porém nesse momento a fonoaudióloga poderia sugerir brincadeiras ao longo da interação.

Segunda sessão

Situação 3 - semi-dirigida com cuidador (mãe)

Foi utilizado o mesmo material da brincadeira livre, porém nesse momento a mãe foi orientada pela fonoaudióloga a brincar com seu filho como quisesse, naturalmente.

Situação 4 - imitação

Incluiu duas etapas: imitação de esquemas gestuais simples e seqüenciais em rotinas familiares. $\mathrm{O}$ adulto apresentava uma ação e falava ao mesmo tempo, para que a criança imitasse em seguida.

As situações de brincadeira livre e semidirigidas duraram 20 minutos (análise dos 15 minutos finais); a imitação foi variável (cerca de 10 minutos).

As tarefas iniciaram no chão, com o adulto e a criança posicionados frente a frente, e a caixa de brinquedos/objetos entre eles. A ordem de realização das atividades foi da menos para a mais dirigida, para evidenciar os comportamentos espontâneos da criança e evitar que a interferência do adulto influenciasse no desempenho posterior.
A análise da habilidade de atenção compartilhada foi realizada para cada situação (adaptação dos comportamentos propostos por Mundy et al. $)^{18}$ :

. alternar: número de vezes que a criança alternou o olhar entre um objeto mecânico ou não, um que estava em suas mãos ou fora de alcance, e a face do adulto;

. apontar: número de vezes que a criança apontou para um objeto próximo ou não de si ou para parte da sala;

. mostrar: número de vezes que a criança estendeu o objeto na face do avaliador ou chamou a atenção deste para algo que estava em suas mãos;

. olhar para ação do adulto: número de vezes que a criança virou a cabeça e olhos em qualquer direção que o adulto apontou, mostrou ou chamou a atenção dela.

Quando ocorreu a combinação de comportamentos, ambos foram pontuados para análise. As respostas foram registradas no protocolo, considerando-se o número de vezes que cada comportamento foi observado em cada situação.

Os comportamentos alternar, apontar e mostrar também foram classificados quando ocorreram por iniciativa da criança (IC - criança utilizou espontaneamente estes comportamentos associados ou não à fala, com o objetivo de compartilhar a atenção) ou se foram em resposta à ação do adulto (RA - adulto chamou a atenção da criança com algum propósito, com uso da fala; gesto; ou ambos, e desencadeou dela uma resposta através dos comportamentos analisados).

O comportamento olhar para ação do adulto considerou somente respostas da criança através das ações/comentários do adulto. Foi verificado se a criança respondeu (SIM) ou deixou de responder (NÃO) quando o adulto chamou sua atenção pela utilização do meio verbal $(\mathrm{V})$, gestual $(\mathrm{G})$ ou verbal e gestual $(\mathrm{V}+\mathrm{G})^{12,19}$.

Foi feito um sorteio aleatório de $50 \%$ dos sujeitos em cada situação para análise por duas fonoaudiólogas com experiência clínica em transtornos do espectro autístico e que estavam familiarizadas com o material, mas desconheciam os dados de identificação e o diagnóstico dos casos. Não foram encontradas diferenças estatisticamente significantes entre estes dois observadores (Prova de Wilcoxon), o que indicou compatibilidade na análise dos dados. 


\section{Resultados}

Os dados obtidos foram pontuados para cada tarefa, e analisados estatisticamente (Prova de Wilcoxon e Prova de Friedman), com nível de significância 0,05 .

A Tabela 1 traz a comparação dos comportamentos alternar, apontar e mostrar nas quatro situações. O alternar predominou de maneira estatisticamente significante em relação ao apontar e mostrar nas quatro situações $(\mathrm{p}<0,001 *)$. Na IC, não foi encontrada diferença estatisticamente significante neste comportamento em nenhuma situação. Na RA, o alternar foi significantemente menos freqüente na brincadeira livre, em relação às situações semidirigidas com terapeuta/cuidador e imitação, que não diferiram entre si.

Quanto ao apontar, não foi encontrada diferença estatisticamente significante na IC nas quatro situações. Na RA, este foi significantemente menos freqüente na imitação comparado às situações semidirigidas com terapeuta/cuidador. A brincadeira livre não se diferenciou estatisticamente das outras situações.

No mostrar, não foi encontrada diferença estatisticamente significante entre as quatro situações na IC. Na RA, este foi significantemente mais freqüente na situação semi-dirigida com terapeuta do que na brincadeira livre e imitação. A situação semi-dirigida com cuidador não se diferenciou estatisticamente das outras situações.

Quanto ao comportamento olhar para ação do adulto (Tabela 2), não foi encontrada diferença estatisticamente significante no uso pelo adulto dos meios $\mathrm{V}$ ou $\mathrm{G}$ nas quatro situações. Já o meio $\mathrm{V}$ $+\mathrm{G}$ foi presente de maneira estatisticamente significante na imitação em relação às situações semi-dirigidas com terapeuta/cuidador. A brincadeira livre não se diferenciou estatisticamente das outras situações.

\section{Discussão}

Nesta pesquisa, considerou-se a ocorrência da atenção compartilhada tanto sob a forma de pedido (chamar a atenção do outro para conseguir algo que deseja), quanto declarativa (direcionar a atenção do outro para algum objeto ou situação) $)^{10,12-13}$.

Será discutida agora a comparação das quatro situações propostas, primeiramente dos comportamentos de alternar, apontar e mostrar, e em seguida do olhar para ação do adulto.
TABELA 1. Comparação entre as quatro situações para os comportamentos alternar, apontar e mostrar.

\begin{tabular}{cc}
\hline Comportamento & $\mathrm{P}$ \\
\hline alternar - iniciativa da criança & 0,346 \\
alternar - resposta à ação do adulto & $<0,001 *$ \\
apontar - iniciativa da criança & 0,520 \\
apontar - resposta à ação do adulto & $0,006 *$ \\
mostrar - iniciativa da criança & 0,065 \\
mostrar - resposta à ação do adulto & $0,013 *$ \\
\hline
\end{tabular}

Legendas: $*$ p-valor: $0,05 \%$

TABELA 2. Comparação entre as quatro situações para o comportamento olhar para a ação do adulto.

\begin{tabular}{|c|c|}
\hline Comportamento & $\mathrm{P}$ \\
\hline $\begin{array}{l}\text { olhar para ação do adulto - verbal } \\
\text { ( } \operatorname{sim} \text { / total) }\end{array}$ & 0,748 \\
\hline $\begin{array}{l}\text { olhar para ação do adulto - gestual } \\
\text { (sim / total) }\end{array}$ & 0,652 \\
\hline $\begin{array}{c}\text { olhar para ação do adulto - verbal + } \\
\text { gestual (sim / total) }\end{array}$ & $\begin{array}{c}0,005 * \text { imitação }>(\text { semi-dirigida } \\
\text { com terapeuta }=\text { semi-dirigida com } \\
\text { cuidador })\end{array}$ \\
\hline
\end{tabular}

Legendas: $*$ p-valor $=0,05 \%$

A princípio, o adulto direciona sua ação de acordo com os interesses da criança, para promover momentos de compartilhamento da atenção. Conforme a criança se desenvolve, passa a chamar a atenção do outro para iniciar os eventos de atenção compartilhada através do olhar, dos gestos e da fala ${ }^{5-6,20-21}$.

Assim, inicialmente a atenção compartilhada ocorre mais em RA, do que por IC. No espectro autístico, existem falhas precoces nestes dois aspectos, sendo a IC ainda mais comprometida ${ }^{12,30}$.

O comportamento alternar predominou sobre os de apontar e mostrar nas quatro situações; não se diferenciou na IC, mas em RA foi menos freqüente na brincadeira livre quando comparado às outras situações. Nesta tarefa, o adulto interferiu o mínimo possível na atividade, fazendo-o predominantemente em resposta à IC.

O comportamento apontar ocorreu menos na imitação, devido à própria característica da tarefa, já que a criança deveria reproduzir as ações a partir do modelo do adulto (raramente incluiu seu uso 
espontâneo ou sua solicitação).

Já o comportamento mostrar, foi mais freqüente em RA na situação semi-dirigida com terapeuta, quando comparado à brincadeira livre (comportamentos espontâneos da criança) e à imitação (reprodução de ações que não incluíram mostrar). Esse contexto facilitou o uso desse gesto pela criança e refletiu o maior compartilhamento da atenção.

O alternar envolve a troca de olhares entre a criança, um objeto/situação e o adulto. Surge entre nove - onze meses e funciona como pré-requisito para a regulação com os gestos de apontar e mostrar, a partir dos onze meses ${ }^{5,21}$.

Assim, o predomínio geral do alternar refletiu uma forma mais inicial de compartilhar atenção, devido aos prejuízos presentes nos sujeitos do espectro autístico, pois não ocorreram mudanças na IC nas diferentes situações.

Em contrapartida, a maioria das crianças aumentou os comportamentos em RA nas situações mais interativas (semi-dirigidas e de imitação). Pesquisas apontaram a IC para compartilhar a atenção como um dos prejuízos mais persistentes no autismo ${ }^{9,13,22}$.

No comportamento olhar para ação do adulto, foi encontrada diferença estatisticamente significante somente em resposta ao meio $\mathrm{V}+\mathrm{G}$ na imitação em relação às situações semi-dirigidas com terapeuta/cuidador, devido ao próprio contexto da atividade.

Alguns autores verificaram que a associação de pistas facilitou o compartilhar da atenção ${ }^{12-19}$. Bruner $^{6}$ já enfatizava que o adulto, ao associar o direcionamento do olhar à fala, estabelece o mecanismo mais eficaz para a ocorrência da atenção compartilhada, que é uma habilidade essencial para o desenvolvimento social e de linguagem e possibilita à criança dividir experiências e emoções.

Neste trabalho, a mãe e a fonoaudióloga interagiram com as crianças, que estavam em terapia há pelo menos seis meses e suas famílias recebiam orientações periódicas para os diversos aspectos do desenvolvimento social e de linguagem-inclusive atenção compartilhada. Estes aspectos são importantes para a ampliação das aquisições obtidas para outros contextos e pessoas ${ }^{4,8,10-11,23-24}$.

Não foram encontradas diferenças estatisticamente significantes no desempenho do grupo nas situações semi-dirigidas com a fonoaudióloga e com a mãe. Provavelmente, ambas foram influenciadas pelo comportamento espontâneo das crianças e procuraram aproveitar as situações de compartilhamento de atenção para estabelecer uma sincronia e corresponder às expectativas delas, dentro das limitações de seus quadros. Esta sincronia foi relatada por Collis e Schaffer ${ }^{25}$ através da interação mãe/bebê, e referida em pesquisas com sujeitos do espectro autístico como essencial para o desenvolvimento social e de linguagem ${ }^{26-27}$.

O uso do material e a adaptação do procedimento da avaliação da maturidade simbólica para verificar a iniciativa e a resposta para atenção compartilhada em sujeitos do espectro autístico foi eficaz e auxiliará na identificação precoce, no diagnóstico diferencial e no prognóstico. Isso porque a atenção compartilhada surge cedo no desenvolvimento típico e, portanto, é possível observar precocemente se existe alguma alteração na manifestação desses comportamentos. Além disso, sendo os prejuízos na atenção compartilhada descritos como precoces e persistentes nesses quadros ${ }^{3-4,28}$, alguns autores observaram diferenças no desempenho de sujeitos do espectro autístico em relação a outros quadros como deficiência mental ${ }^{18}$ e distúrbio de desenvolvimento de linguagem ${ }^{23}$, nos quais observaram pior desempenho dos sujeitos do espectro autístico. Finalmente, algumas pesquisas indicaram que dentre os quadros do espectro autístico, existem diferenças na manifestação dos comportamentos de atenção compartilhada, que a longo prazo influenciam no desenvolvimento de linguagem ${ }^{10,18,24}$.

A brincadeira promove momentos agradáveis de compartilhamento da atenção, e permite a observação espontânea da criança e dos efeitos da intervenção do adulto, através de situações contextualizadas e interessantes para o sujeito do espectro autístico ${ }^{9,12,29-31}$.

$\mathrm{Na}$ prática clínica, a prioridade seria a comparação entre as situações de brincadeira livre (observação mais espontânea) e semi-dirigida com um adulto familiar (observação das modificações geradas por um interlocutor preparado para corresponder às expectativas da criança), as quais promovem momentos naturais de interação e permitem a observação das iniciativas e respostas da criança com material padronizado. A prova de Imitação não deve ser considerada isoladamente como parâmetro de análise.

Os achados reforçam as observações de outros pesquisadores, pois apesar de a atenção compartilhada estar severamente comprometida e servir como um marcador precoce nos sujeitos do espectro autístico, ela não está totalmente ausente. Esta habilidade é extremamente importante para o diagnóstico e intervenção precoce e específica baseados neste aspecto $9,13,18,26$. 


\section{Conclusão}

A partir das situações de interação propostas, foi possível verificar que os sujeitos do espectro autístico apresentaram a habilidade de compartilhar

\section{Referências Bibliográficas}

1. Wing L. Asperger`s syndrome: a clinical account. Psychol Med. 1981;21:115-29.

2. Perissinoto J. Diagnóstico de linguagem em crianças com transtornos do espectro autístico. In: Ferreira L P, Befi-Lopes D M, Limongi S C O. Tratado de Fonoaudiologia. São Paulo: Roca, 2004. p. 933-40.

3. Kanner L. Autistic disturbances of affective contact. Nervous Child. 1943;12:57-69.

4. Perissinoto J. Processo de Evolução da Criança com Autismo. In: Perissinoto J. Conhecimentos Essenciais para Atender bem a Criança com Autismo. São Paulo: Pulso, 2003. p. 23-27.

5. Carpenter M, Nagell K, Tomasello M. Social cognition, joint attention, and communicative competence from 9 to 15 months of age. Monogr Soc Res Child Dev.1998;63(4):1143.

6. Bruner J. Child's talk: learning to use language. London: Norton \& Company - New York, 1983. cap. 2,4.

7. Mundy P, Crowson M. Joint attention and early social communication: implications for research on intervention with autism. J Autism Dev Disord. 1997; 27(1):653-76.

8. American Speech-Language-Hearing Association. Guidelines for Speech-Language Pathologists in Diagnosis, Assessment, and Treatment of Autism Spectrum Disorders Across Life Span. 2006. Available from: http:// www.asha.org/members/deskref journal/deskref/default.

9. Sullivan M, Finelli J, Marvin A, Garrett-Mayer E, Bauman M, Landa, R. Response to joint attention in toddlers at risk for autism spectrum disorder: a prospective study. J Autism Dev Disord. 2007:37:37-48.

10. Whalen C, Schreibman L. Joint attention training for children with autism using behavior modification procedures. J Child Psychol Psychiat. 2003;44(3):456-68.

11. Kasari C, Freeman S, Paparella T. Joint attetion and symbolic play in young children with autism: a randomized controlled intervention study. J Child Psychol Psychiatry.2006:47(6):611-20.

12. Leekam SR, Ramsden CA. Dyadic orienting and joint attention in preschool children with autism. J Autism Dev Disord. 2006;36(2):185-97.

13. Warreyn P, Roeyers H, Van Wetswinkel U, De Groote I. Temporal coordination of joint attention behavior in preschoolers with autism spectrum disorder. J Autism Dev Disord. 2007;37:501-12.

14. American Psychiatric Association. Manual Diagnóstico e Estatístico de Transtornos Mentais - DSM IV-TR. Porto Alegre: Artes Médicas; 2002.

15. Organização Mundial da Saúde. Classificação estatística internacional de doenças e problemas relacionados à saúde: CID 10. $10^{\text {a }}$ ed. São Paulo: Edusp; 1993. a atenção e modificaram seus comportamentos positivamente a partir da interferência de um interlocutor.

16. Befi-Lopes DM, Takiuchi N, Araújo K. Avaliação da maturidade simbólica nas alterações do desenvolvimento da linguagem. J Bras Fonoaudiol. 2000;1(3):6-15.

17. Takiuchi N. Relações entre Maturidade Simbólica e Desenvolvimento Lingüístico em crianças com Distúrbios Específicos de Desenvolvimento da Linguagem [tese]. São Paulo: Universidade de São Paulo; 2006.

18. Mundy P, Sigman M, Kasari C. A Longitudinal Study of Joint Attention and Language Development in Autistic Children. J Autism Dev Disord.1990;20(1):115-27.

19. Leekam SR, Hunnisett E, Moore C. Targets and cues: gaze-following in children with autism. Child Psychol Psychiatry Allied Discipl. 1998;39(7):951-62.

20. Iverson J, Capirci O, Longobardi E, Caselli MC. Gesturing in mother-child interactions. Cognitive Development. 1999;14:57-75.

21. Capone NC, McGregor KK. Gesture development: a review for clinical and research practices. J Speech Lang Hear Res. 2004;47:173-86.

22. Cardoso C, Fernandes FDM. Relação entre os aspectos sócio cognitivos e perfil funcional da comunicação em um grupo de adolescentes do espectro autístico. Pró-Fono Rev. Atual. Cient. 2006;18(1):89-98.

23. McArthur D, Adamson LB. Joint Attention in Preverbal Children: Autism and Developmental Language Disorder. J Autism Dev Disord. 1996;26(5):481-95.

24. Koegel LK. Interventions to facilitate communication in Autism. J Autism Dev Disord. 2000;30(5):383-91.

25. Collis GM, Schaffer HR. Synchronization of visual attention in mother-infant pairs. J Child Psycho. Psychiatry. 1975;16(4):315-20.

26. Siller M, Sigman M. The behaviors of parents of children with autism predict the subsequent development of their children's communication. J Autism Dev Disord. 2002;32(2):77-89.

27. Miilher LP, Fernandes FDM. Análise das funções comunicativas expressas por terapeutas e pacientes do espectro autístico. Pró-Fono Rev. Atual. Cient. 2006;18(3):239-48.

28. Bosa C. Atenção compartilhada e identificação precoce no autismo. Psicol Reflex e Crit. 2002;15(1):77-88.

29. Tamanaha AC. Intervenção na Linguagem da Criança com Autismo. In: Perissinoto J. Conhecimentos Essenciais para Atender bem a Criança com Autismo. São Paulo: Pulso, 2003. p. 55-60.

30. Warreyn P, Roevers H, De Groote I. Early social communicative behaviours of preschoolers with autism spectrum disorder during interaction with their mothers. Autism. 2005;9(4):342-61.

31. Stanley GC, Konstantareas MM. Symbolic play in children with autism spectrum disorder. J Autism Dev Disord. 2007;37(7):1215-23. 